\title{
CORRECTION
}

\section{Author Correction: The natural product 4,10- aromadendranediol induces neuritogenesis in neuronal cells in vitro through activation of the ERK pathway}

Sai Chang ${ }^{1}$, Wen-chen Ruan ${ }^{1}$, Ya-zhou Xu ${ }^{1,2}$, Yun-jie Wang ${ }^{1}$, Jie Pang ${ }^{3}$, Lu-yong Zhang ${ }^{1,4}$, Hong Liao ${ }^{1}$ and Tao Pang ${ }^{1,2,4}$

Acta Pharmacologica Sinica (2020) 41:730; https://doi.org/10.1038/s41401-019-0339-0

Correction to: Acta Pharmacologica Sinica https://doi.org/10.1038/ aps.2016.115, published online 14 November 2016

The authors apologize that one image in Fig. 2a appeared incorrectly. The representative image for the positive control "NGF" group in Fig. $2 \mathrm{a}$ is the same as that for the " $10 \mu \mathrm{mol} / \mathrm{L}$ ARDD

a
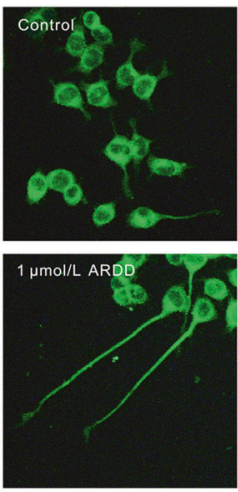

C
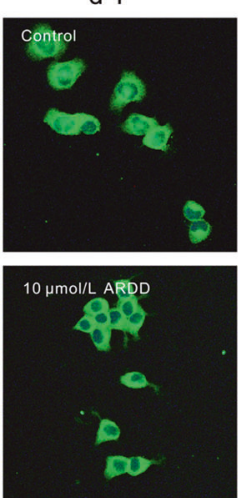
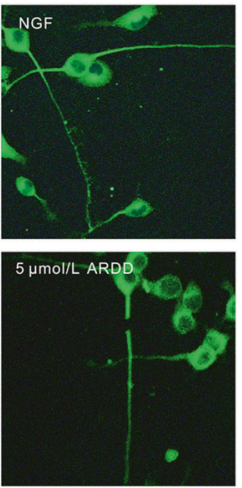

d 2
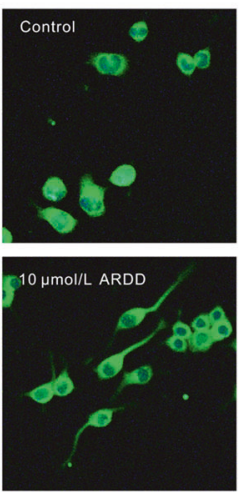

MAP-2/Hoechst
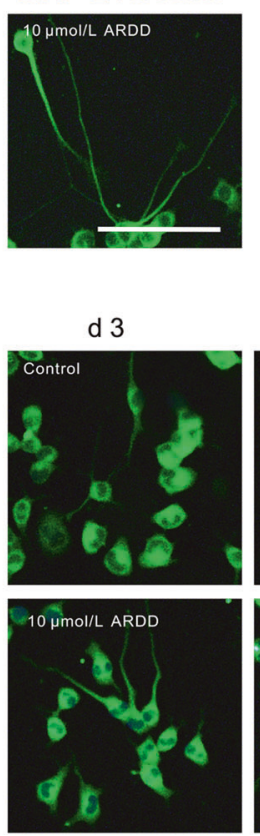

in D4" group in Fig. 2c. The correct picture for the positive control "NGF" group in Fig. 2a is given. This correction does not affect the conclusions of the original article. We apologize again for any inconvenience caused.

The correct Fig. 2 is shown below. b

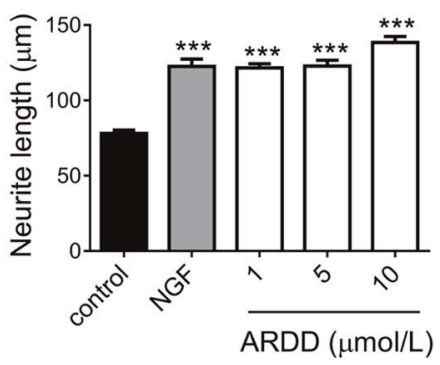

d

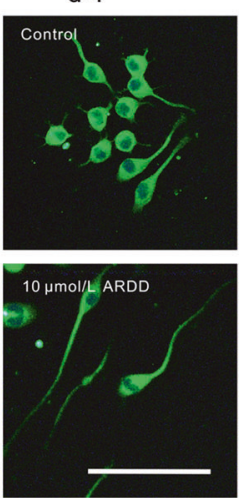

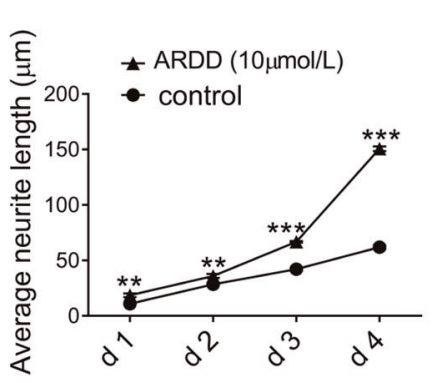

Fig. 2 ARDD induces neurite outgrowth of N1E115 cells. a Effects of different doses of ARDD on neurite outgrowth of N1E115 cells at day 4. The bar represents $100 \mu \mathrm{m}$. b Statistics of average neurite length in above groups at day 4 . The data are expressed as the mean \pm SEM $(n=$ $80-100)$. ${ }^{* *} P<0.001$ vs control group. c Time-course manner of $10 \mu \mathrm{mol} / \mathrm{L}$ ARDD promoting neurite outgrowth of N1E115 cells. The bar represents $100 \mu \mathrm{m}$. d Statistics of average neurite length at different days. The data are expressed as the mean \pm SEM $(n=80-100)$. ${ }^{* *} P<0.01$, $* * * P<0.001$ vs control group.

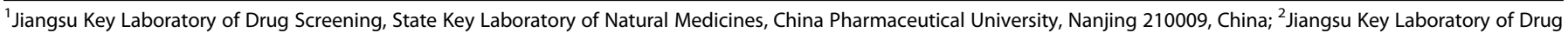

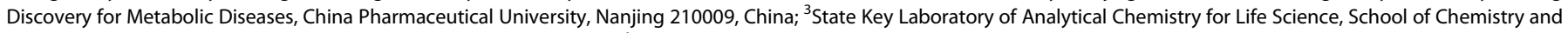

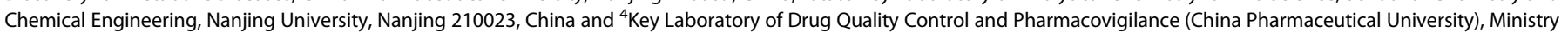
of Education, Nanjing 210009, China

Correspondence: Hong Liao (liaohong56@hotmail.com) or Tao Pang (tpang@cpu.edu.cn)

Published online: 21 February 2020 